\title{
QUEEN'S
UNIVERSITY
BELFAST
}

\section{Using mesh-geometry relationships to transfer analysis models between CAE tools}

Tierney, C., Nolan, D., Robinson, T., \& Armstrong, C. (2015). Using mesh-geometry relationships to transfer analysis models between CAE tools. Engineering With Computers, 31(3), 465-481.

https://doi.org/10.1007/s00366-014-0377-7

\section{Published in:}

Engineering With Computers

\section{Document Version:}

Peer reviewed version

\section{Queen's University Belfast - Research Portal:}

Link to publication record in Queen's University Belfast Research Portal

\section{Publisher rights}

Copyright 2014 Springer-Verlag London

The final publication is available at Springer via http://dx.doi.org/10.1007/s00366-014-0377-7

\section{General rights}

Copyright for the publications made accessible via the Queen's University Belfast Research Portal is retained by the author(s) and / or other copyright owners and it is a condition of accessing these publications that users recognise and abide by the legal requirements associated with these rights.

Take down policy

The Research Portal is Queen's institutional repository that provides access to Queen's research output. Every effort has been made to ensure that content in the Research Portal does not infringe any person's rights, or applicable UK laws. If you discover content in the Research Portal that you believe breaches copyright or violates any law, please contact openaccess@qub.ac.uk. 


\title{
Using mesh-geometry relationships to transfer analysis models between CAE tools
}

\author{
Christopher Tierney, Declan Nolan, Trevor Robinson and Cecil Armstrong \\ School of Mechanical and Aerospace Engineering, Queen's University \\ Belfast, BT9 5AH ctierney06@qub.ac.uk
}

\begin{abstract}
Integrating analysis and design models is a complex task due to differences between the models and the architectures of the toolsets used to create them. This complexity is increased with the use of many different tools for specific tasks during an analysis process. In this work various design and analysis models are linked throughout the design lifecycle, allowing them to be moved between packages in a way not currently available. Three technologies named Cellular Modeling, Virtual Topology and Equivalencing are combined to demonstrate how different finite element meshes generated on abstract analysis geometries can be linked to their original geometry. Cellular models allow interfaces between adjacent cells to be extracted and exploited to transfer analysis attributes such as mesh associativity or boundary conditions between equivalent model representations. Virtual Topology descriptions used for geometry clean-up operations are explicitly stored so they can be reused by downstream applications. Establishing the equivalence relationships between models enables analysts to utilize multiple packages for specialist tasks without worrying about compatibility issues or substantial rework.
\end{abstract}

Key Words. Mesh-geometry ownership, CAD / CAE integration, Cellular Modeling, Virtual Topology, Equivalence

\section{Introduction}

The introduction of Computer Aided Design (CAD) and Computer Aided Engineering (CAE) tools has had a major impact on the Product Development Process. Designs can be developed and tested in the virtual environment, reducing the need for expensive prototypes. Computational analysis methods like finite element analysis (FEA) have progressed from validation and failure verification tools to design and concept verification tools, resulting in them being employed earlier in design cycles where analysis results drive the design process [15]. Consequently, the capabilities of modern analysis tools are rapidly increasing, along with the complexity of 
the analyses being undertaken. This has led to more detailed analyses being performed at earlier stages of design processes. Aerospace companies undertake multi-level, multi-disciplinary analyses of components throughout the design process. The multi-disciplinary analyses allow for a more accurate assessment of the overall behavior of a system, but leads to a significant burden when preparing the different analysis models.

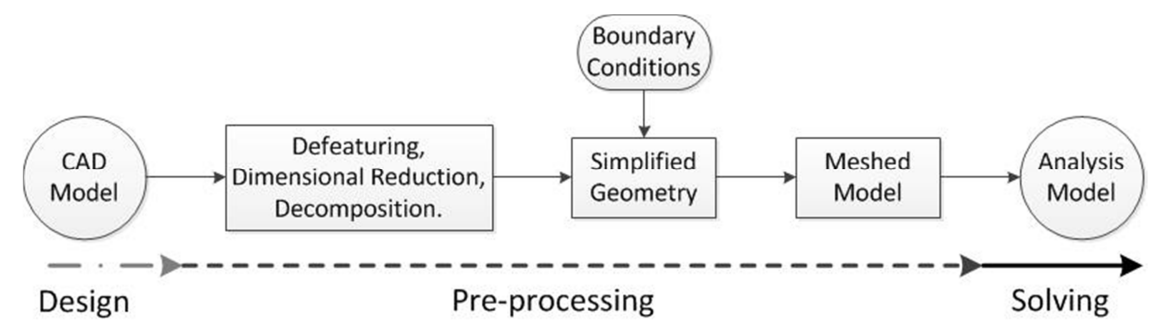

Fig. 1. Common analysis process using current methods

In many analysis cycles there are numerous pre-processing steps required to enable geometric domains to be more adequately meshed, Fig. 1. Preprocessing steps may include model simplifications where manufacturing or aesthetic design details which are assumed to have no simulation significance are removed [18]. The pre-processing steps tend to vary depending on analysis requirements and the stage of the design process, e.g. details that are significant for a stress analysis may be irrelevant for a modal analysis. In other circumstances different geometric decompositions may be required for analyzing different load cases for a product so that high stress areas can be accurately represented. With multiple decompositions of the same CAD model required for different analysis tasks, it is essential that bi-directional links exist between equivalent models [1]. These links are necessary to ensure that results can be exchanged between different analysis models at varying levels of fidelity. This is especially true for coupled multi-disciplinary analyses where the output from one simulation acts as the input to another e.g. thermo-mechanical analysis.

In reality industrial companies utilize numerous specialist tools for analysis activities. One such example is aero-elastic analyses where distinct solvers are often utilized in order to achieve as accurate a solution as possible. The tools employed depend heavily on the task being performed, the type of physics involved, the stage of the design process and the complexity of the component or assembly to be analyzed [5]. Early in a design process it may be appropriate to use an automatically generated unstructured tetrahedral (tet) mesh to gain an initial insight into the performance of the product. However, later in the design process it may be desirable to use multiple different analysis packages with specific hexahedral (hex) meshing capabilities. This can result in multiple analysis models existing for a given component with no robust link between them and no link back 
to the original design geometry. Furthermore, the fact these equivalent analysis representations are often generated by different people, and within distinct analysis pre-processing packages, adds significantly to the complexity of the $\mathrm{CAD} / \mathrm{CAE}$ integration problem.

Solving computational analysis problems requires a fit-for-purpose finite element mesh to be generated on the abstract analysis geometry, along with any applicable analysis attributes such as boundary conditions, loading and material properties. The application of boundary conditions for large assemblies like whole engine thermo-mechanical models can be a tedious and time consuming task due to the vast number of physical interactions present in the assembly models. This process is frequently complicated by the necessity to generate different abstract analysis models for both thermal and structural domains. For example, a thermal analysis may be carried out using a tet mesh automatically generated on an abstract analysis model. However, the abstract analysis model may need to be simplified further in order to obtain a suitable hex mesh for a large-deformation structural analysis. Without a robust method to transfer analysis information between packages the application of boundary conditions often has to be repeated many times during the lifecycle of a design. It is shown in this work that the ability to link meshes from various analysis geometries back to the original CAD model (and by extension each other) reduces rework in terms of setting up the analysis model, even when they are generated within different analysis packages.

Mesh-geometry ownership is the relationship between individual mesh entities and their parent B-Rep entity. During mesh generation modern packages automatically impose mesh-geometry ownership, allowing applied boundary conditions to be transferred to the corresponding nodes and elements of the mesh [2]. This process is more convenient than applying boundary conditions to individual mesh entities, which can be cumbersome for even the simplest of models. For the purposes of assembling the finite element matrices, boundary conditions on the B-Rep entities are internally converted to equivalent nodal 'loads' or imposed 'displacements'. Therefore, CAD model updates or mesh modifications can be made without having to reapply loads. Using current tools it is not always the case that mesh-geometry ownership can be successfully transferred from the package in which it was created to a different downstream package, especially when the models are of varying levels of abstraction or fidelity. This makes repetitive manual operations essential in order to successfully recreate the mesh-geometry ownership at other stages in the design process, which is highly undesirable for situations such as mesh adaption where frequent global and/or local mesh refinements may be performed. Therefore, robust links between equivalent model representations are required.

In this work, recording the equivalent relationships between design and analysis models enables finite element meshes created on abstract analysis 
geometries to be fully associated with the original design model, regardless of the packages within which they were created. This enables analysis attributes like element properties and boundary conditions to be transferred between different packages and analysis models at various levels of fidelity, without compromising the model integrity. This gives an analyst the freedom to select the desired tools for specific aspects of the analysis process without having to worry about compatibility issues or any substantial rework involved in transferring and rebuilding analysis models. Releasing the analyst from time consuming manual rework allows more consideration to be given to the analysis being performed.

\section{Three technologies for linking design and analysis models}

This work describes how three technologies named Cellular Modeling, Virtual Topology and Equivalencing are used to manage and manipulate the topology of geometric design and analysis models, enabling them to be linked, independent of any underlying CAD or CAE package [19]. These technologies are not new by themselves, but their combination for $\mathrm{CAD} / \mathrm{CAE}$ integration in the manner described is novel. In this section a brief description of the three technologies is provided.

\subsection{Cellular Modeling}

Cellular representations are defined as non-manifold representations of both positive (solid) and negative (void) regions [3]. In manifold representations any point on the boundary of a solid region has a neighborhood homeomorphic to a 2-dimensional disk [20]. Geometric representations that are not manifold are referred to as non-manifold. An example of a non-manifold condition is a face which bounds two distinct volume cells. The face and its bounding entities are shared between the two volume cells. Using cellular models means these interfaces can be identified and utilized to define boundary conditions between adjacent components. Fig. 2 (b) shows the interfaces in a non-manifold cellular representation.

Cellular representations have been used in assembly mesh generation, where non-manifold topological entities between interacting components provide suitable interfaces for conformal meshing [21]. Conformal meshing is considered the most accurate form of connectivity between volume cells with compatible element types and is often used instead of contact elements or constraint equations. At non-manifold interfaces shared faces are meshed before the volume mesh is created, resulting in shared nodes at the common interface. In other work Sypkens-Smit and Bronsvoort [17] 
used cellular modeling representations for remeshing feature models. Cells in the cellular model are related to their parent features so they can be tracked after model updates, allowing boundaries to be compared so that local remeshing can be efficiently achieved. Lee et al. [6] introduced a method for CAD model simplification using cellular representations. In this approach each feature in a design model is explicitly defined as a volumetric cell in the global cellular model. Cells below a certain threshold volume can be suppressed to achieve the desired abstracted model for different applications.

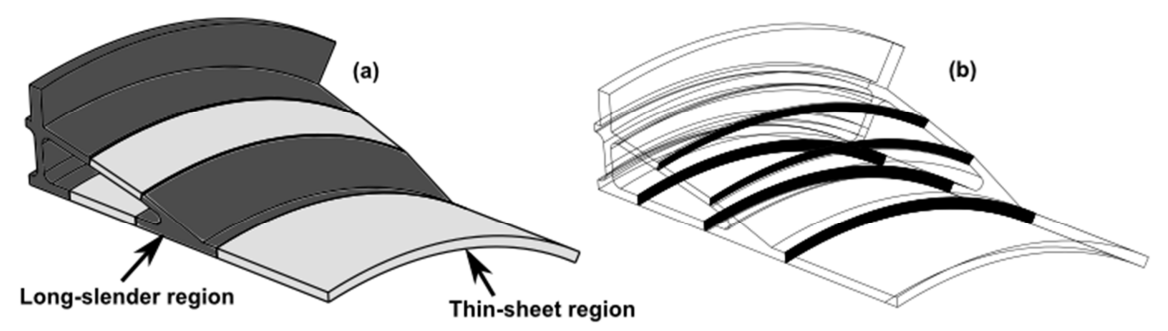

Fig. 2 Non-manifold cellular representations: (a) Casing component partitioned into thin-sheet and long-slender regions, (b) Highlighted interfaces between interacting cells

In this work a region in a non-manifold representation is referred to as a cell. Cells can have any manifold dimension, i.e. volumes, faces, edges and vertices are all considered as regions of space bounded by other cells. Cellular models represent the entire design space subdivided into regions with specific analysis significance. Fig. 2 (a) shows a model that has been partitioned into sub-regions to support downstream meshing, more details of which can be found in $[8,13]$. The analysis significance of each cell can be used to automatically determine how to manage the interfaces between adjacent cells. For instance, mesh connections, such as multi-point constraints (MPCs) or rigid links, can be used at interfaces between cells that are meshed with incompatible mesh types. Handling the interfaces between components offers the chance to automate many manual preprocessing tasks, particularly when used with the analyst's 'Simulation Intent' as described in [10].

Assigning attributes to volume cells enables lower level topological attributes to be automatically managed and used to build relationships between equivalent models. Every cell in the cellular decomposition contains information specifying its origin in relation to the design model. The origin of a cell can be used along with the interfaces between interacting cells to link design and analysis models at various levels of fidelity. In situations where multiple cells have the same originating cell it is possible to use Virtual Topology to create associations between the different cells. 


\subsection{Virtual Topology}

Virtual Topology was introduced by Sheffer et al. [14] as a technique for preparing CAD models for analysis purposes. It allows simplifications to be made on a model without having to directly modify the geometry, which may introduce even more changes from the original model. Virtual Topology operations use real topological entities called Hosts in order to create virtual entities. Virtual superset entities represent the combination of multiple adjacent entities of the same manifold dimension, while virtual subset entities represent a partial section of another entity. Parasite entities are used to split a higher dimensional topological entity into several subset entities e.g. a parasite face is used to partition a volume to simplify the geometric model for meshing applications. These virtual entities are generated using the virtual topology operators described below.

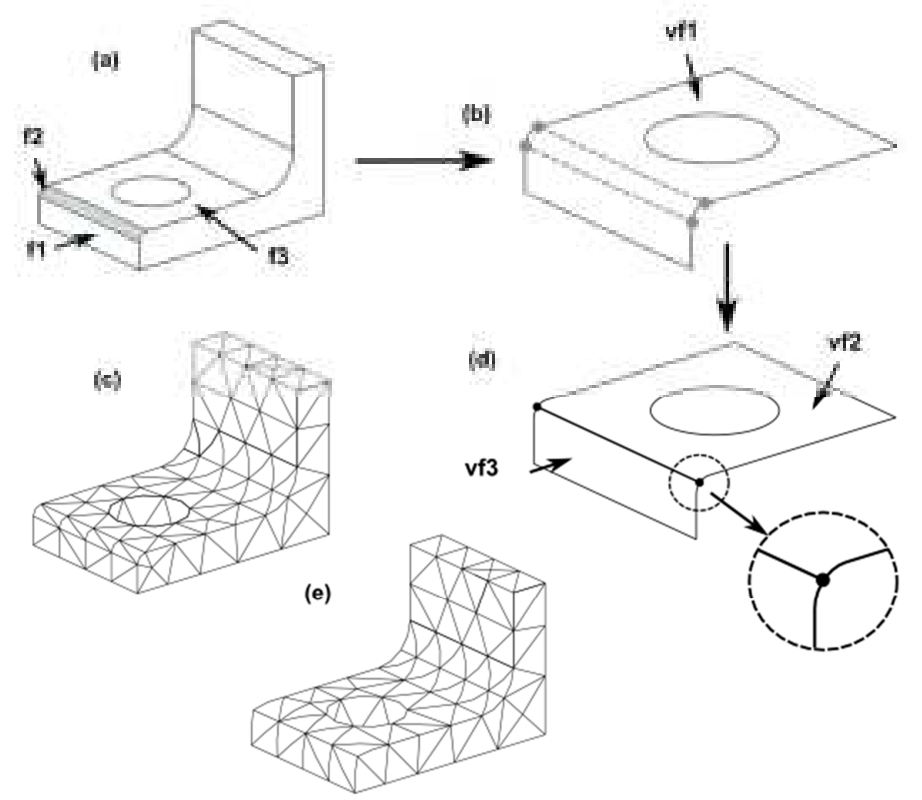

Fig. 3. Virtual Topology geometry clean-up used to collapse a face to an edge. (a) Model with small blend feature, (b) Superset face, (c) Coarse mesh on original model, (d) Subset faces, (e) Coarse mesh on abstract analysis model

The two main Virtual Topology operators utilized in this work are the merge and split operators. Using the merge operator adjacent entities of the same manifold dimension are merged into a single virtual superset entity by ignoring the common boundary between them, for example the dashed edges and highlighted vertices in Fig.3 (b). The split operator is used to partition a single host entity into virtual subset entities. The host entity can be a real or virtual topological entity, such as the superset face that has been partitioned in Fig. 3 (d). Virtual parasite entities are used to partition 
the boundary of the host entity to create the virtual subsets. Sheffer [14] uses the construction operator to generate virtual parasite entities by defining their bounding entities, i.e. a parasite edge is created by defining its bounding vertices.

One application of Virtual Topology is geometry clean-up for mesh generation, as shown in Fig. 3. Small features like blend faces, highlighted grey in Fig. 3 (a). Small features like blend or sliver faces provide problems to mesh generation algorithms as nodes are usually distributed along all bounding entities in a model. When faces are small in comparison to the target element size, poorly shaped elements are created, Fig. 3 (c), which can have an adverse effect on the accuracy and efficiency of an analysis. Actual abstraction of the blend feature may vary depending on the application or the preference of the analyst. One method is to merge the blend face with its adjacent faces and to partition the resulting virtual superset face, a process which is illustrated in Fig. 3 (b) and (d). These Virtual Topology procedures effectively collapse the blend face to an edge and are used to modify the topological connectivity of the blend feature, so as to achieve a mesh coarser than the blend size, Fig. 3 (e). Even though the topology of the face has been altered, its geometric attributes remain intact. This is beneficial in situations where a feature needs to be retained from an analysis perspective, but its topology can still be modified to facilitate the creation of block topologies for semi-structured hex meshing.

Many analysis or mesh generation packages currently have Virtual Topology capabilities. Some packages make these Virtual Topology decisions internally without reporting the details to the analyst, making it difficult for the analyst to determine where it has been used and therefore to interrogate or modify the Virtual Topology. Once Virtual Topology has been used to simplify a model for meshing, certain packages do not allow the simplified geometry to be exported. These issues make it difficult to reuse the Virtual Topology generated and counteract the desired automation of the analysis process. Virtual Topology operations are preprocessing steps taken to produce fit-for-purpose analysis geometry and should be robustly stored as such.

The ability to interrogate the Virtual Topology enables the Virtual Topology relationships (the linkages between host entities and virtual entities) to be stored and ultimately accessed by other downstream applications. Section 3 describes a general procedure for extracting the VT in situations where it cannot be directly extracted from the CAE solution it was generated within. Identifying and storing Virtual Topology decisions within an external data structure enables the relationships to be accessed and utilized by other CAE packages regardless of the Virtual Topology capabilities of the downstream client, i.e. once Virtual Topology relationships have been identified and stored the Virtual Topology does not need to be regenerated in another package. In addition, these relationships ena- 
ble multiple analysis decompositions to be linked to each other by using the appropriate non-manifold interfaces to identify Virtual Topology relationships. Once analysis geometries have been linked to the original geometry, meshes generated on the abstract analysis models can be linked back to the original model and, by extension, to each other. In this work the manufacturing detailed design model is referred to as the original model. Having multiple meshes associated with the same geometry is a significant advantage of this approach, especially where it may be necessary to transfer results between the distinct meshes. Whilst the actual mapping of results is not covered in this work the links between the respective meshes are identified and stored in an accessible manner.

\subsection{Equivalencing}

Different analysis models and therefore different meshes are required for different applications during the design process. The analysis models can differ due to the stage of the analysis or even the type of analysis being performed. Early in design processes simpler analysis models representing fewer details and with fewer degrees of freedom may be utilized as approximate results are often acceptable, provided they are returned quickly. Analysis complexity normally increases as the design evolves. Different meshes are required to solve different physics problems and to get more detailed results.

In this work it is considered that as a mesh is a representation of a domain, all meshes of that domain can be considered equivalent to it and each other. The representative example, in Fig. 4, shows various analysis models that are considered equivalent as they represent the same component. For example, early in the design process a pinion shaft may be represented using two 1-dimensional beam elements with different crosssectional properties, Fig. 4 (a). A coarse tet mesh may be required for a modal analysis, Fig. 4 (b) and a structured hex mesh may be required at a later stage for an impact analysis, Fig. 4 (c). During detailed design stages a hybrid mesh including tooth geometry may be necessary for a stress analysis, Fig. 4 (d). While these different representations are adequate for their specific purpose, in this work equivalence relationships are determined between equivalent cells in the model. For example, the pinion body is represented as a beam element in Fig. 4 (a), a tet meshed or hex meshed cylinder cell in Fig. 4 (b) and (c) and a tet mesh of a detailed geometry including gear teeth in Fig. 4 (d). The end vertices of the beam elements are considered equivalent to planar faces of the pinion head in other representations. Similarly, relationships between the simple gear and detailed gear cells can be determined by exploiting the links to the dimensionally reduced representation. For example, once the end vertices of the 1D idealization representing the pinion head are identified as equivalent to the pla- 
nar faces of the pinion head in the more detailed representations, all residual faces bounding the pinion head are considered equivalent to the wire edge of the beam element. The relationships between the collection of tooth faces and the cylindrical (smoothed) face of the pinion head are determined by exploiting the links between the detailed geometries and the equivalent 1D idealization. In situations where equivalent relationships are not available through links to an idealized representation it is possible to utilize the non-manifold combination of the cells, where non-manifold faces represent interactions between cells, to identify the equivalence dependencies. Virtual Topology is used to manage the dependencies between edges faces or volumes cells which have been merged, or partitioned, for analysis purposes without modifying the geometric representation. This is opposed to Equivalencing, which is used to manage the dependencies between distinct geometric or analysis models which exist due to the design evolution, or due to modifications such as feature suppression or dimensional reduction.

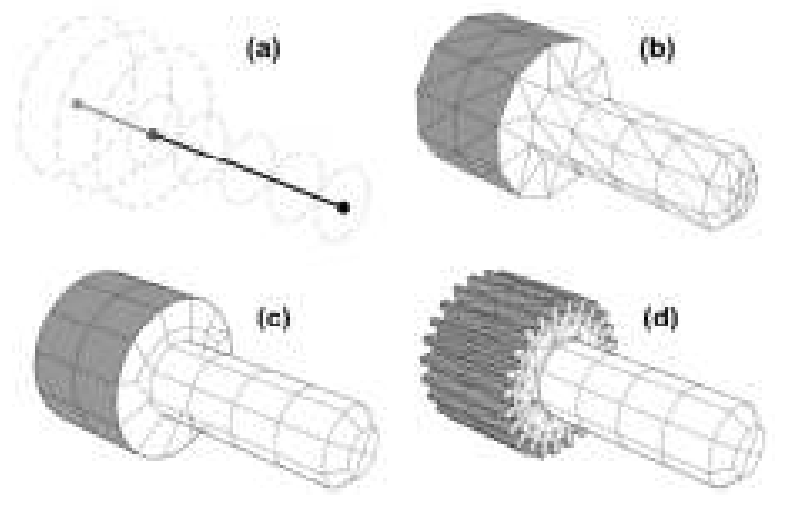

Fig. 4. A simple pinion shaft with different meshes applied for specific tasks during a design process: (a) Beam elements with different cross-sectional properties, (b) Linear tet mesh, (c) Structured hex mesh, (d) Hybrid mesh.

By establishing the equivalences between individual analysis geometries and the original model it is possible to transfer analysis attributes and results between all models generated for that domain. The actual transfer of analysis attributes, such as loading, is not covered in this work and would require the transformation of boundary conditions between equivalent representations, i.e. the dimensional reduction of boundary conditions as described by Donaghy [4]. The extent of the transformation is dictated by the shape difference between the equivalent representations. 


\section{Robust mesh transfer process}

In many CAE tools boundary conditions are applied to topological entities in the simplified geometric model, Fig. 1. If analysis and design models are not connected then boundary conditions need to be reapplied for each subsequent analysis model. This could be extremely costly where a design is updated frequently. Using the approach described here boundary conditions can be defined on the original design geometry without having to worry about any downstream idealizations that may occur, Fig. 5.

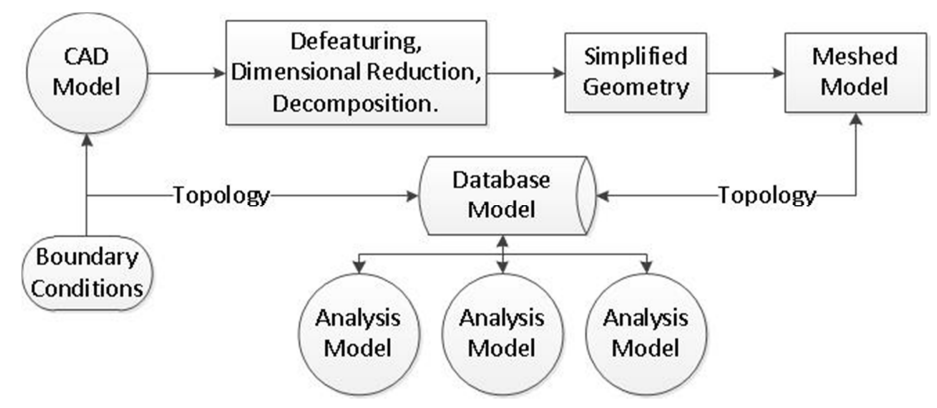

Fig. 5. Analysis process using the master database to link various representations.

Once fit-for-purpose meshes have been generated, the simplified geometric model is no longer required to achieve the analysis solution. Storing equivalent links between the simplified and original models allows meshgeometry ownership to be transferred between the models. Therefore, boundary conditions assigned to the original model can be automatically transferred to the mesh of the idealized model before it is solved. This results in integrated design and analysis capabilities that can have major benefits in large collaborative projects with many distributed partners. Different departments and sub-contractors are assigned specific tasks within the analysis process. Each partner may prefer to use their toolsets of choice without having to consider how this may affect downstream collaboration, or without sharing any information about how the models were generated to protect intellectual property. Example tasks which may be integrated include model simplification, meshing, assignment of boundary conditions etc. Integrating the models produced and required by the different analysis tools ensures tighter integration of the entire design and analysis process.

In order to transfer various finite element attributes between different models, residing in different packages, it is essential that the various models are robustly linked $[7,16]$. The ability to relate different meshes back to the same design model provides tighter integration between the disciplines. In order to link these models a simple data structure has been developed which is independent of any underlying CAD or CAE package. The data structure has been implemented in the form of a relational data- 
base whose entity relation diagram is detailed in Fig. 6, [19]. Its purpose is not to replace existing data structures used to represent models in CAD and CAE systems, rather it is used to store the non-manifold topology of the cellular model for the product being designed, and also the different approximations and analysis models that represent it. All equivalent decompositions of the product are stored regardless of their dimension or the tools used to create them. The database acts as a master model for linking these equivalent representations. The advantage of using a non-manifold model is that non-manifold interfaces are used, along with Virtual Topology and equivalence information to integrate the different models. A complete description of the data structure and its use for analysis applications is available in [19].

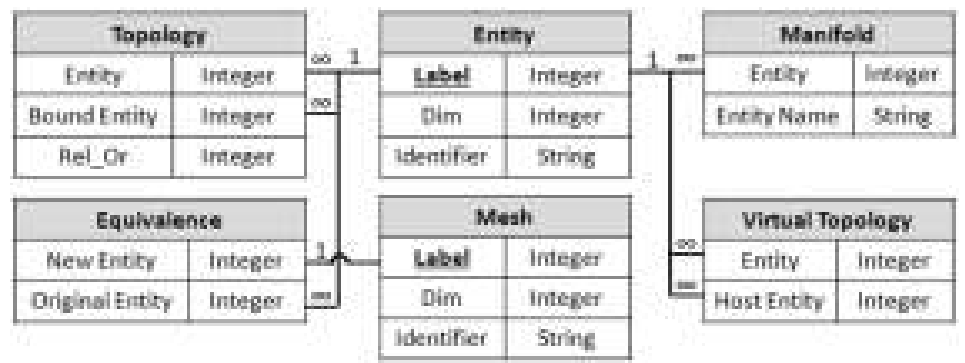

Fig. 6. Database entity-relation diagram.

Once the analysis geometry has been created its non-manifold topology can be stored in the data structure, Fig. 6. The topology is stored independently of the packages used to create the model and in a generic format that makes it accessible by any CAD or CAE package. The main topological entities (vertices, edges, faces and volumes) are represented in the Entity relation using their unique Identifier, which is a point within the boundary of the entity. The Identifier enables robust identification of entities between various packages. This is in comparison to naming attributes that may go missing, change or cannot be applied to a topological entity. The topological connectivity of a model is stored in the Topology relation of the data structure, where each row defines a cell, one of its bounding entities and the relative orientation of the two (e.g. whether a surface normal points into or out of a body). Bi-directional links exist between the topology in the data structure and different design and analysis models using the Virtual Topology relation and an Identifier. This is stored as a String in the entity table but actually defines a point contained within boundary of the entity.

The topology of various decompositions of the same component can be stored concurrently in the data structure, along with any mesh-geometry ownership relationships. A simple example is introduced Fig. 7 to describe the relationships between equivalent decompositions. By identifying the 
links between the original, Fig. 7 (a), and simplified geometries, Fig. 7 (b), it is possible to link their respective meshes. The Virtual Topology relation of Fig. 6 is used to store the link between the equivalent models. Superset edge 've1' is created by merging edges 'e1', 'e2' and 'e3', which are stored as its host entities, Fig. 7 (e). Virtual superset face 'vfl' is stored in the same manner for faces ' $\mathrm{fl}$ ', ' $\mathrm{f} 2$ ' and ' $\mathrm{f3}$ '. Linking the original and virtual models enables their meshes to be linked by manipulating their meshgeometry relationships. Therefore, results may be transferred between the different models. Consider the original geometry in Fig. 7 (a) and where a mesh for a thermal analysis has been created to calculate the temperatures on each face, Fig. 7 (c). A fine mesh can be used for a thermal analysis as nodes have only one degree of freedom and the analysis is relatively inexpensive. Once temperatures have been calculated Virtual Topology relationships are used to link them to the simplified model in Fig. 7 (b). To achieve this, the collection of element faces of the thermal analysis mesh are related back to their parent topological faces (' $\mathrm{fl}$ ', ' $\mathrm{f} 2$ ' and ' $\mathrm{f3}$ '). These topological faces are linked to the virtual face in the simplified model and by extension to the coarse mesh, Fig. 7 (d), applied to the virtual face. Once the links have been determined temperature values can be mapped and interpolated between source and target meshes in order to execute a structural analysis. The interpolation between meshes is not explored in this paper. The important point is that the meshes used for different analyses can be linked using the Virtual Topology for the two equivalent geometries.

(a)

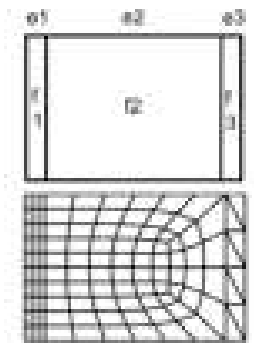

(b)

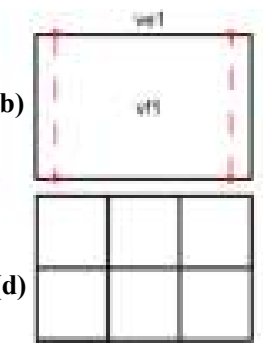

\begin{tabular}{cc}
\hline Entity & Host Entity \\
\hline ve1 & e1 \\
ve1 & e2 \\
ve1 & e3 \\
vf1 & f1 \\
vf1 & f2 \\
vf1 & f3 \\
\hline
\end{tabular}

(e)

Fig. 7. Linking different decompositions: (a) Original geometry, (b) Simplified geometry, (c) Fine mesh on original geometry, (d) Coarse mesh on simplified geometry, (e) Virtual Topology relationships.

In this work the goal is to establish and store relationships between the topological entities in equivalent design and analysis representations. Virtual Topology is used to store the relationships between different representations when a one-to-one correspondence does not exist between related entities. These cells have to be defined as supersets or subsets of different cells. Equivalent relationships are stored when the same region of the design space is represented at different levels of fidelity. Once these relation- 
ships have been defined, analysis attributes can be transferred between models at various levels of fidelity.

\section{Identifying Virtual Topology relationships}

Automated Virtual Topology tools available in commercial CAE packages can be used for geometry clean-up. They operate by identifying small features that may hinder the mesh generation process and merge them with adjacent larger features in the model, without altering the actual CAD geometry. Entities are merged by ignoring their common bounding entities, i.e. common edges are ignored to merge adjacent faces. To enable Virtual Topology operations to be reused in other downstream applications it is necessary to establish relationships between virtual entities and their host entities so they can be stored in the database. Since many CAE tools do not report the details of Virtual Topology operations, it is necessary to identify the entities in the original model to which it has been applied, (i.e. the host entities). The two types of virtual entities to be identified are superset and subset entities, where superset and subset entities are created by merge and split operations respectively. The relevant Virtual Topology relationships can be extracted by finding either the entities that have been ignored, where a superset has been created, or the new entities (parasites) that have been introduced, where subset entities have been created.

\subsection{Virtual Topology for geometry clean-up}

Virtual merge operations are predominantly used during geometry cleanup operations to remove troublesome features like sliver faces. Virtual subsets may be required to partition an entity for load application, mesh control, or as a precursor to the creation of a superset entity, where it may be necessary to merge subset entities. Once created, the identification of both virtual subsets and superset relationships will be described in this section.

Fig. 8 shows a simple example where both merge and split operators are used in order to generate an appropriate abstract analysis model. The smaller blend in the model may be ignored as it is below the target element size or to simplify the domain prior to volume decomposition for meshing (section 4.2). Topology comparisons between the original model and the abstract analysis model have been used to identify the virtual topology relationships generated as a result of merge or split operations. Virtual subset relationships are identified by finding entities that exist in the abstract model but not in the original model. Conversely, entities that do not exist in the abstract model as opposed to the original model have been used to 
link virtual superset entities to their host entities. Having already stored the topology of the original model in the data structure it is relatively straightforward to identify topological differences between the models. The ability to maintain multiple representations at once in the data structure provides the functionality to use virtual entities as the input to further virtual operations.
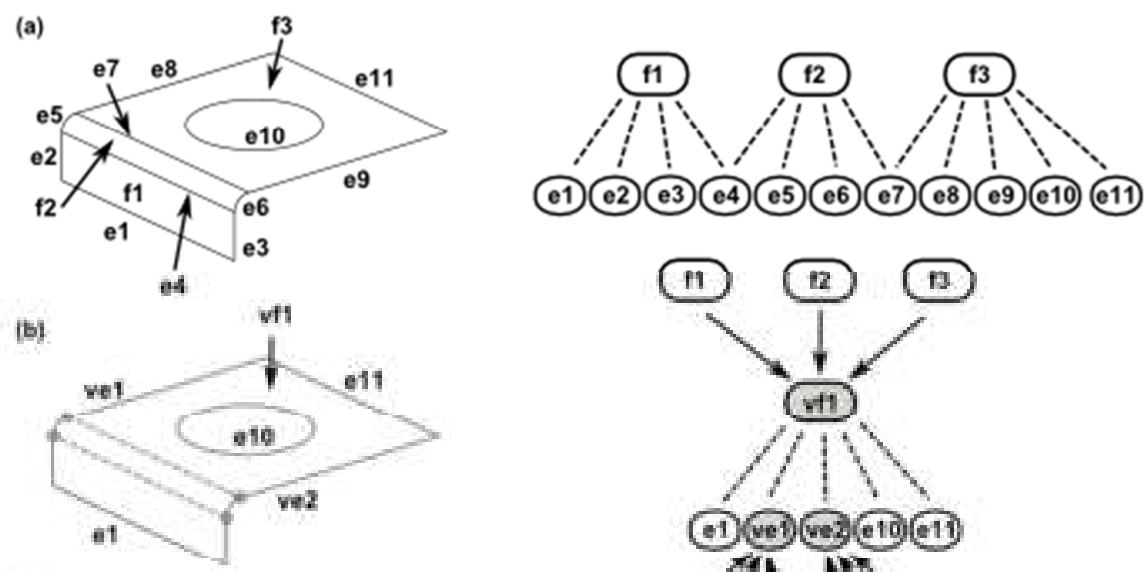

(c)
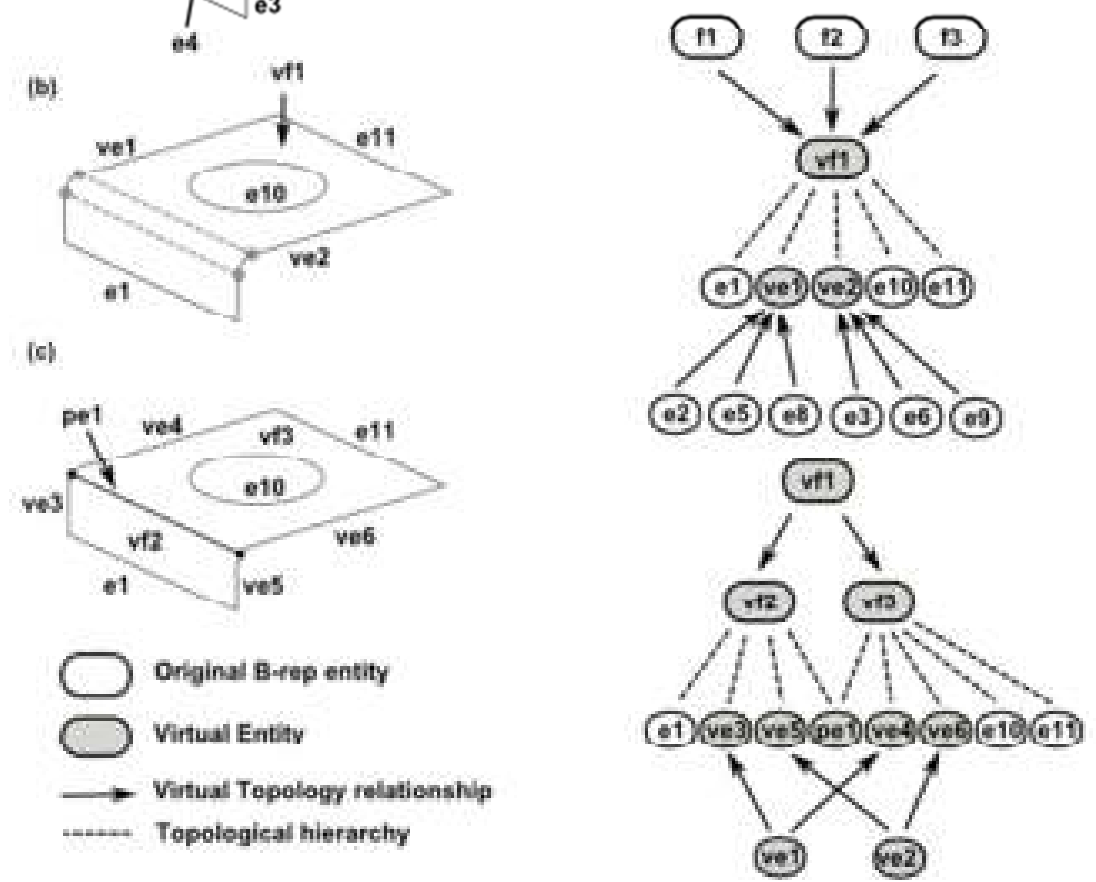

Fig. 8. Virtual Topology merge and split operations used to collapse a blend face to an edge: (a) Original faces and bounding edges, (b) Resulting superset face and edges after merge operation, (c) Virtual subset faces created by split operation. Virtual Topology and Topological dependencies for each model are illustrated on the right hand side.

Entities that have been ignored in the abstract model represent the common boundary, dashed grey edges and bounding vertices in Fig. 8 (b), between adjacent entities that have been merged together to create a virtual superset entity. The first step compares the positions of vertices in the original model to those in the abstract model. Ignored vertices are those in the original model that do not exist in the abstract model, vertices bounding edges 
'e4' and 'e7' in Fig. 8 (a). Once the ignored vertices have been identified their bounded edges are returned by interrogating the topological connectivity which has previously been stored in the data structure. Connected pairs of bounded edges are grouped together to ensure the correct relationship is defined between the virtual superset and all host edges, Fig. 8 (b) where edge 've1' is the superset of host edges 'e2', 'e5' and 'e 8 '. These host edges have been merged together by removing their common boundaries to create the virtual superset edge. The topology of the superset edge is automatically created in the data structure by finding the unique bounding entities of the host entities, i.e. the unshared bounding entities. In the same manner the relationship between any superset faces and their underlying host faces have been determined. Once all vertices in the abstract model have been identified, the topological connectivity in the database is used to identify any edges. Ignored edges can then be identified and their bounded faces in the original model automatically returned from the data structure. Therefore, the new superset face is automatically linked to its host faces and the relationship stored in the Virtual Topology relation. For example, virtual superset face 'vfl' in Fig. 8 (b) is the union of faces ' $\mathrm{fl}$ ', ' $\mathrm{f2}$ ' and ' $\mathrm{f3}$ ' in the original model, Fig. 8 (a). The topology of the virtual superset entity is stored in the Topology relation of the data structure. It is seen in Fig. 8 (b) that superset face 'vfl' is bounded by original edge 'el', 'e10' and 'e11' along with virtual edges 've1' and 've2'. The topological adjacency information is automatically updated for all entities bounded by virtual entities.

After identifying virtual supersets relationships it is possible to identify virtual subsets and their single host entity. Entities present in the abstract model but not the original model are classified as parasite splitting entities. Topological entities that are bounded by these parasite entities represent the virtual subset entities that have been created due to a split operation. For example, the black highlighted edge in Fig. 8 (c) is identified as a parasite edge. It is visible from the Virtual Topology relationships in Fig. 8 (c) that parasite edge 'pe1' is not stored in the Virtual Topology relation but is easily identified as the common boundary between virtual subset faces. The bounded faces of this parasite edge are categorized as virtual subset faces, 'vf2' and 'vf3' in Fig. 8 (c). The link between the subset faces and their host face, which has been partitioned by the parasite edge, is established by finding edges which are not shared by any of the subset faces, the 'uncommon' edges of the subset faces. The existing topology in the data structure is interrogated to find the host face bounded by these uncommon edges. In this case the host face is the superset face 'vfl'. The Virtual Topology dependencies in Fig. 8 (c) show that virtual entities can be referenced as by new virtual entities, i.e. superset face ' $\mathrm{vfl}$ ' is partitioned into virtual subsets 'vf2' and 'vf3', while virtual superset edge 've1' is split to form subset edges 've3' and 've4'. Uncommon edges that have been used 
in previous merge operations are replaced by the virtual superset edge, allowing the correct face to be identified using the topological interrogations described.

Virtual Topology relationships are stored in the data structure along with the topology of the virtual entities. Storing these relationships in the data structure creates the links between the original and abstract models and enables them to be reused by downstream applications. Therefore, once a mesh has been generated it can be linked to a different model in a different package without having to recreate any virtual entities. This is a major benefit of storing the Virtual Topology in an accessible manner,

\subsection{Virtual Topology for linking equivalent geometries}

Defeaturing, dimensional reduction and decomposition tools [8,9, and 18] are commonly used to create idealized analysis models which are less computationally expensive. There are occasions where a model may be decomposed or partitioned into idealized sub-regions in order to meet the specific meshing requirements of an analyst. A requirement for many analyses is the creation of a mesh comprised of only hexahedral elements as they are more computational efficient due to their structured layout, i.e. hex elements are desirable for highly non-linear transient events as they allow larger time steps for the explicit solver. Robust automated hex mesh generation is still a largely unsolved problem. Geometry decomposition methods are used to create hex meshable sub-regions.
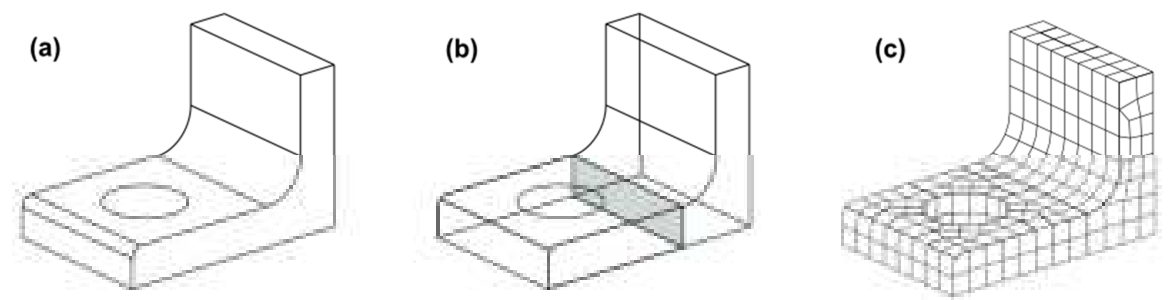

Fig. 9. Decomposition of a simple component into hex meshable sub-regions: (a) Original component; (b) Decomposed model with parasite face highlighted; (c) Hex meshed component.

An example model is shown in Fig. 9 (a) where the simple component cannot be automatically hex meshed by many commercial CAE packages. Some CAE packages are able to automatically subdivide a model such as this into hex meshable sub-regions, Fig. 9 (b), which can then be automatically hex meshed, Fig. 9 (c), in that or a different package. The model in Fig. 9 (b) has the same geometry clean-up operations applied to the fillet as described in the previous section. The hex mesh is generated for the decomposed cells by creating a quad mesh on the appropriate source face and 
sweeping it through the volume, as the wall faces facilitate mapped meshing. This effectively simplifies the hex meshing problem into the $2 \mathrm{D}$ domain for sweepable regions.

With the objective of relating the mesh generated on the simplified geometric model to the original model, it is necessary to establish the correct equivalences between the models. Relationships between host entities in the original model are defined as the union between all subset entities in the decomposed model. This process is the same as described for the subset entities in the previous section, where parasite entities are identified in the sub-divided model. The manifold dimension of parasite entities is one less than the entity they partition, i.e. vertices are used to split edges, edges are used to split faces and faces are used to split volumes, Fig. 10. Therefore, the bounded entities of a parasite entity define the subsets, while the uncommon boundaries between the subset entities returns the bounding entities of the host entity that has been partitioned.

(a)

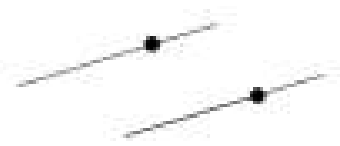

(b)

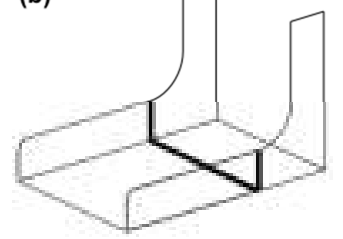

(c)

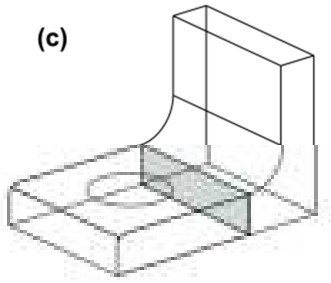

Fig. 10. Linking the original and sub-divided models: (a) Parasite vertices (highlighted) used to split original edges, (b) Parasite edges (highlighted) used to split original faces, (c) Parasite face (highlighted) used to split an original volume.

\section{Mesh-geometry ownership}

In this work a mesh is considered to be an equivalent representation of the geometry it represents. Mesh generation processes in commercial packages position nodes and elements onto topological entities in the geometric model. The relationship between a mesh entity and the topological entity it represents is stored in the Equivalence relation of the data structure. The topological entity referenced by a mesh entity is referred as the parent while the mesh entity is referred as the child entity. This information is referred to as parentage relationships. Mesh entities are stored in the 'New Entity' attribute and their parents in the 'Original Entity' attribute. Mesh entities can have only one distinct topological entity as their parent, while topological entities may have multiple mesh entities linked to them. This is shown in Fig. 6 where the entity-relation diagram of the data structure imposes the constraint that mesh entities can only appear once in the "New Entity' attribute of the equivalence relation. This constraint ensures a one- 
to-one relationship is maintained between the mesh entity and its parent topological entity. However, topological entities can appear many times in the 'Original Entity' attribute as they can have multiple child entities. These relationships are used to successfully transfer a mesh between models at various levels of fidelity.

\subsection{Assigning mesh parentage relationships}

Mesh entities include nodes, element edges, element faces and elements. Different relationships may exist between mesh entities and their parent topological entities. Nodes may have B-Rep vertices, edges, faces or volumes as their distinct parent entity. Element edges and faces can have BRep edges and faces as their respective parents if they lie on topological boundaries. Elements have B-Rep parent entities equivalent to their dimensionality. For example, solid elements will have B-Rep volume cells as their parent while shell elements will have B-Rep faces as their parent entity.

Storing relationships between all mesh entities and their parent B-Rep entities would introduce redundant relationships that would complicate the transfer process. For example, element edges and faces are not equivalent to topological edges and faces if they lie inside the boundary of a region, which would require constant manipulation between equivalent relationships. Loads and boundary conditions, such as pressure loads, are assigned either directly as nodal values or to element faces or edges. The choice of method is dependent on the analysis package. Therefore, parentage information may, or may not, be required for certain mesh entities depending on the package. However, it is certain for all packages that loads and boundary conditions are ultimately represented on nodes and elements of the finite element mesh before an analysis is executed [11]. Due to these issues it has been decided to store parentage information for only node and element mesh entities. If certain packages require the use of element edges and faces for boundary condition application they can be derived from the element connectivity and equivalence relationships, which are readily available from the mesh file and the data structure. Correspondence is retained between mesh entities in the data structure and the mesh file by utilizing the same node and element numbers within each format.

Each B-Rep topological entity in the model is queried to find its mesh child nodes. To ensure each node has only one distinct parent only nodes that do not lie on the bounding entities of a B-Rep entity acquire that entity as their parent. The correct nodal parentage relationships are formed using a bottom-up approach, shown in Fig. 11. Topological entities with the smallest manifold dimension are addressed first for the example where a hex mesh is generated on the cuboid, Fig. 11 (b). Firstly, all vertices in the model are interrogated to find their child node, highlighted black in Fig. 11 
(c). Once the associativity for these vertices has been identified their nodes cannot have another parent entity assigned, despite the fact that they may lie on other B-Rep entities. This is shown in Fig. 11 (d) where the edges are interrogated to find their child nodes which are highlighted black. Only nodes that lie within the bounding vertices of an edge are assigned as children of the edge. Therefore, it follows that B-Rep faces and volumes are assigned as the parent of any child nodes that do not lie on their bounding entities. As a result of the merging process each node is related to only one parent topological entity. Defining the parentage relationships is more straightforward. Each element in the mesh is directly related to a distinct topological parent entity, whose dimensionalities must match one another, i.e. solid, shell and 1D elements have B-Rep volumes, faces and edges as their respective parents.
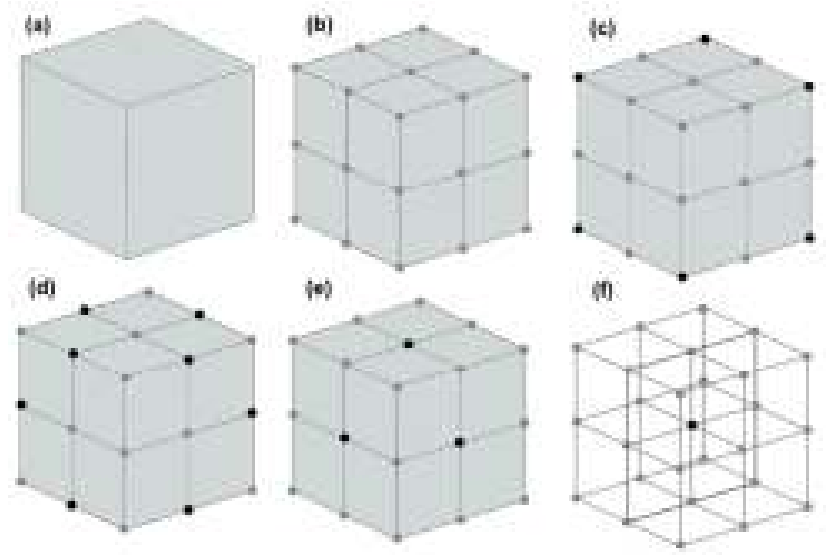

Fig. 11. Nodal B-Rep parentage relationships; (a) Cuboid component, (b) Cuboid meshed with hex elements, (c) Vertex parents, (d) Edge parents, (e) Face parents, (f) Volume parents.

\subsection{Transferring mesh entities between equivalent models}

In previous sections it was described how Virtual Topology relationships were used to link different equivalent models. These relationships are stored in the data structure along with well-defined mesh-geometry parentage information. This allows the links between equivalent models to be exploited so that mesh associations can be robustly transferred. Transferring full mesh associativity between models ensures boundary conditions do not need to be re-applied for different models.

In this work the mesh is generated on the decomposed model and subsequently associated to the original model. Mesh entities with a virtual entity as their parent topological entity are automatically related back to the host entity. Virtual entities include virtual subset, superset and parasite entities. 
Nodes lying on subset edges, faces or volumes, highlighted grey in Fig. 12 (a), are assigned the original edges, faces and volumes, Fig. 12 (b) as their parent entity. Mesh nodes with parasite entities as their parent entity are assigned as the host entity of the parasite entity as their parent. Parasite entities are identified as the common boundary between virtual subset entities. The parent host entity will normally have a manifold dimension of one more than the parasite entity. For example, the highlighted nodes in Fig. 12 (c) have parasite entities as their parent entity. Their equivalent original parent entities are shown in Fig. 12 (b). The node with a parasite vertex as its parent entity is assigned the host entity of the parasite vertex as its parent, which is the original edge. Similarly, the nodes with a parent parasite edge or face have host faces or volumes as their respective parents in the original model. The same process is used to transfer the ownership of elements between models. Elements have a parent topological entity of the same manifold dimension as the element dimensionality and will therefore only have virtual subsets or supersets as their parent entity, never parasite entities.

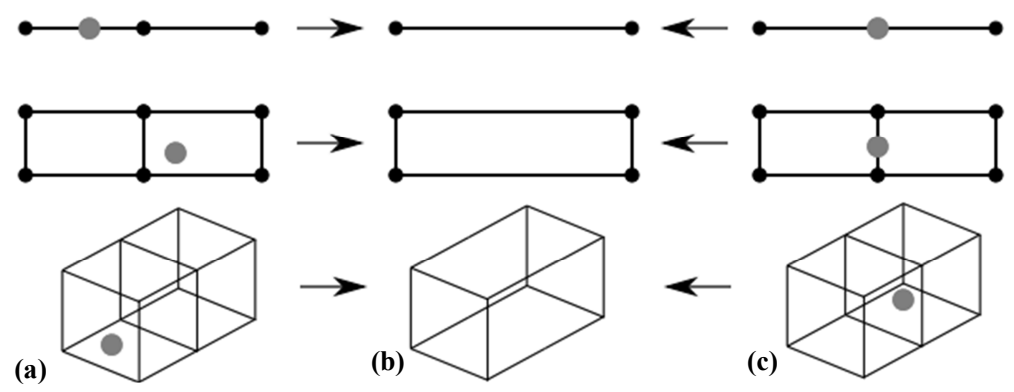

Fig. 12. (a) Nodes with virtual subset entities as their parent, (b) The parent host entity of nodes with parent subsets or parasite entities, (c) Nodes with parent parasite entities.

The transfer of mesh entities between equivalent models is carried out automatically using pre-built SQL queries on the data structure. These queries are sufficient to transfer the associativity for mesh entities with virtual subset or parasite entities as their parent. This is due to the fact that the mesh associations are transferred from a subset or parasite to a single referenced host entity. However, in situations where nodes lie on a virtual superset entity there are multiple host entities referenced. This is shown in Fig. 13 where nodes generated on the superset face, Fig. 13 (b), are related to each individual host face. These relationships are identified at the mesh generation phase using geometric queries to locate nodal positions on the original host faces, Fig. 13 (c). 

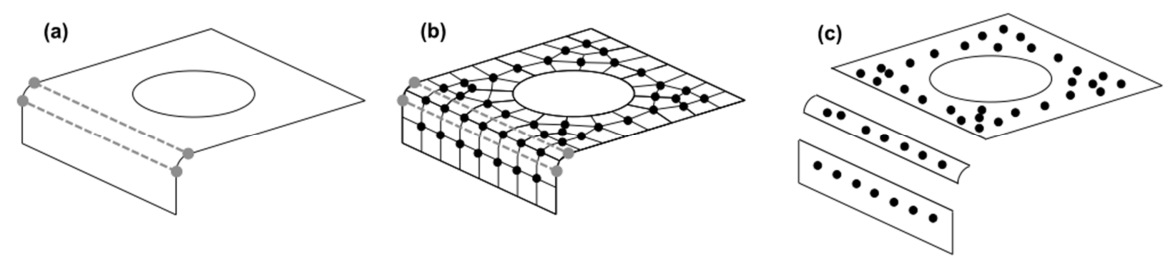

Fig. 13. Linking node with virtual superset parents to their original entity: (a) Superset face with ignored edges dashed, (b) Mesh generated on superset face, (c) Nodes linked back to original host entities.

\section{Contribution towards an integrated design process}

This section demonstrates the automatic implementation of the procedures described in Sections 4 and 5 to transfer a mesh generated on an idealized analysis model within Abaqus to the original design model within CADFix. Automatically determining the dependencies between the equivalent idealized and original models enables the mesh to be transferred between them. Abaqus is utilized for mesh generation and CADFix for creating the input file for analysis, including boundary conditions applied to entities in the original design model. The process described here is required because the mesh is created on a model with a different topology than the original model, which was created in Siemens NX, Fig. 14 (a). This means that any analysis attributes such as loading or boundary conditions defined in CADFix on the topology of the original model need to be transferred to the mesh generated on the abstract analysis topology, Fig. 14 (b). This is currently a manual process and can be time consuming to achieve for complex models. The procedures presented here allow multiple different packages to be used to create suitable analysis geometry and meshes whilst maintaining the links between them and therefore back to analysis attributes applied to any model.

In this example the original model, Fig. 14 (a) has been decomposed into hex-meshable regions using the tools described in $[8,13]$. Once the decomposed model, Fig. 14 (b), has been created it is imported into Abaqus. The choice of tool used to arrive at the subdivided analysis geometry, or destination package (Abaqus for meshing and CADFix to generate the analysis input file), does not restrict the process described in this paper. After importing the analysis model into Abaqus a non-manifold representation is created using the Abaqus Boolean Union tool. The non-manifold model is interrogated and its topology is extracted and stored in the database. Abaqus has an automatic Virtual Topology tool which identifies faces and edges to be merged based on a set of input geometric parameters. The default parameters of this tool are sufficient to remove the unwanted sliver faces in the test model. The entities that have been ignored can be identified in Abaqus, but extra functionality would be necessary to deter- 
mine the exact virtual and host relationships. Therefore, the generic procedure described in Section 4 was used to identify the virtual superset and subset entities, where the decomposed volume cells are treated as subsets of an original cell. Virtual Topology relationships are stored in the database so they can be accessed and interrogated by downstream CAE packages.

The current procedure for identifying Virtual Topology relationships is limited to virtual superset and subset entities. It is often the case that preprocessing packages utilize more exotic Virtual Topology operations (i.e. for collapsing small angles) or even geometry repair and clean-up tools (such as remove or tweak) that actually modify the geometry. Currently, this work would rely on the CAE package to provide access to the more bespoke Virtual Topology operations to enable the relationships to the Brep topology to be retrieved. It is anticipated that once the benefits of exploiting the Virtual Topology for CAD-CAE integration have been demonstrated CAE vendors will be more susceptible to providing direct access to Virtual Topology relationships. Direct geometric modification has not been addressed in this paper as it is the aim to demonstrate that analysis model preparation can be carried out at the Virtual Topology level. More research needs to be carried out to make this a reality, especially for geometry repair.
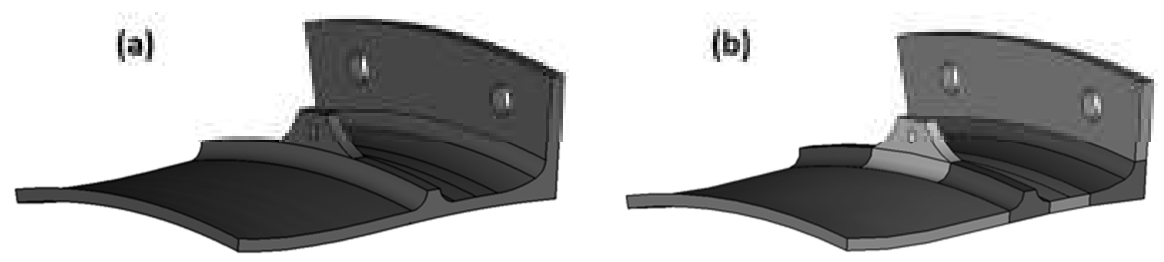

Fig. 14. Domain decomposition: (a) Original geometry, (b) Subdivided analysis geometry.

Abaqus was selected for this demonstration as it can automatically select suitable meshing strategies to apply to certain cells, i.e. a non-manifold representation with multiple interacting cells can be automatically meshed with conformal meshes at the interface. After creating the mesh, all nodes and elements are stored in the Equivalence relation of the database along with their parent B-Rep entities, Section 5. This is achieved by using Abaqus queries to find the nodes belonging to each vertex, edge, face and volume in the non-manifold model. During this process an orphan mesh file is automatically created. The structure of the mesh file is manipulated to suit the target package. For example, element codes are changed to suit the target system, i.e. a 20 node hex element is assigned an element code of C3D20R within Abaqus and a HE20 element code in CADFix. Node and elements in the orphan mesh file are linked to their parent entities in 
the database. This process extracts the mesh parentage information for the mesh generated on the idealized model within Abaqus, Fig. 15 (a).
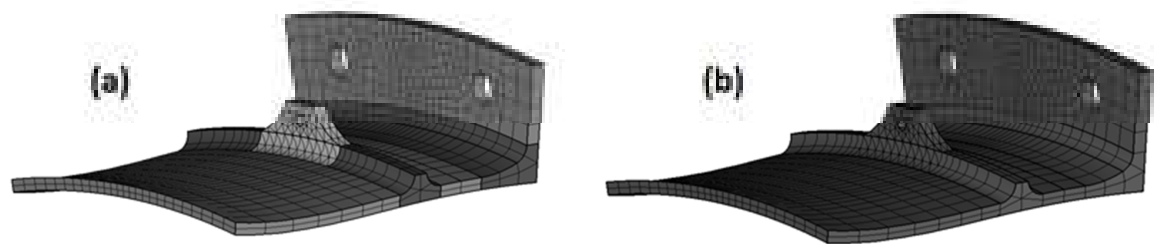

Fig. 15. Mesh transferred between equivalent representations: (a) Mesh generated on sub-divided model, (b) Mesh transferred to original model with full associativity.

Topology comparisons are made to store the Virtual Topology relationships necessary to link the equivalent models. All entities in the data structure are automatically tagged so they can be accessed by the different interacting packages. These relationships allow the topology of the decomposed model in the data structure to be linked to the original model in CADFix, allowing mesh-geometry ownership to be transferred between the two models. This results in the mesh being transferred to the original model representation, Fig. 15 (b), with full associativity between mesh entities and their parent topological entities in the original model. Mesh entities having either parasite or subset entities as their B-Rep parent are linked to the equivalent host entity, Section 5. This completes the transfer of mesh-geometry ownership between models at different levels of fidelity. Analysis attributes assigned to topological entities in the original model can be automatically applied to the correct (equivalent) mesh entities, allowing analysis input files to be generated.

Finally, the analysis attributes applied to a model can be transferred between a master design model and multiple equivalent analysis models, Fig. 16. Cellular Modelling, Section 2.1, provides a method to manage models consisting of cells of different analysis significance. Cells may include fluid and structural domains. The non-manifold nature of the cellular representation allows solid-fluid interfaces to be easily extracted for boundary condition application. Using the Virtual Topology and Equivalence information described in this paper these boundary conditions can be transferred between models at various levels of fidelity. This includes dimensionally reduced analysis models. Relationships between different models are stored in a central, generic data structure that is accessible by all CAD / CAE packages, Fig. 16. Automated tools have been developed to show how analysis attirbutes, such as pressure loads and mesh entities, can be transferred between equivalent model representations. These tools have been tested with Siemens NX, Abaqus, CADFix and Parasolid. 


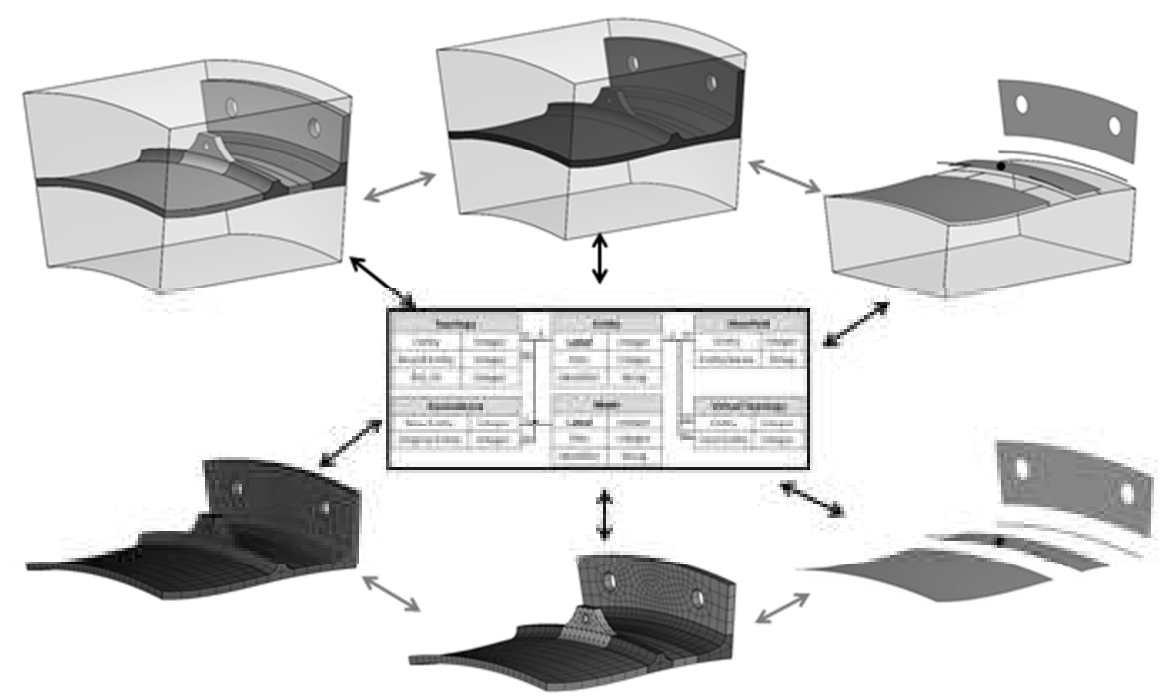

Fig. 16. Linking multiple analysis models using Cellular Modelling, Virtual Topology and Equivalencing maintained in a central data structure.

\section{Discussion}

This paper introduces a method to transfer finite element meshes at different levels of fidelity between different packages by identifying and storing the links between equivalent geometries. The underlying theme is to track the equivalences between the original design model, analysis geometries at various levels of detail and abstraction, and the finite element meshes generated on them. The automated tool described in the previous section enables different packages to be selected based upon their strengths for specific analysis processes but does not restrict the subsequent movement of the analysis model. For the example shown, Abaqus was selected for its meshing capabilities and CADFix was selected as it can provide the input file for different analysis packages. The mesh generated in Abaqus on simplified analysis geometry has been automatically transferred to the original model in CADFix using the automated tool.

Virtual subset and superset relationships are explicitly stored in order to link abstract analysis models generated as a result of geometry clean-up or decomposition procedures. Subset relationships are identified by finding the uncommon boundary between the subset faces bounded by a parasite entity. Superset host entities are those bounded by entities that have been ignored. Mesh entities can be transferred to an original design model using the virtual subset and superset information stored in the data structure. Only the mesh needs transferred between packages and not the analysis ge- 
ometry, which is required only for the initial mesh generation. Therefore, this limits the number of times analysis geometry has to be transferred between packages and avoids possible topological inconsistencies arising due to modeler tolerance etc.

(a)

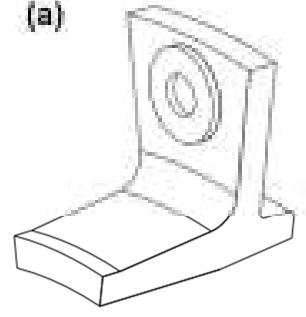

(b)

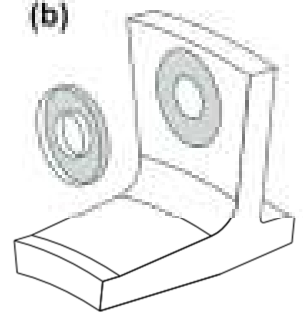

(c)

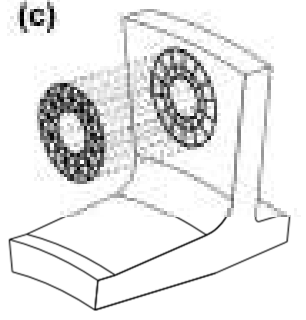

Fig. 17. Transferring mesh attributes: (a) Original manifold assembly, (b) Cellular model with imprinted non-manifold interface highlighted, (c) Dissimilar meshes linked on original manifold model in (a).

Example Fig. 17 (a) shows a manifold flange and washer assembly. The creation of a non-manifold cellular model automatically imprints interacting flange and washer faces on each other, highlighted faces in Fig. 17 (b). The components are offset in Fig, 17 (b) and (c) for clarity. Storing the imprinted entities as subsets of original host entities enables dissimilar meshes to be linked to one another without having to transfer the imprinted analysis geometry between packages, Fig. 17 (c). This provides a robust solution as abstract models cannot be exported from many packages, especially where Virtual Topology operations have been used. Groups of nodes on the non-manifold interface can be connected using non-manifold interface information in the data structure and finding child nodes for each interacting face. Entities are tracked within the data structure to allow nonmanifold relationships to be made use of from within a manifold environment, i.e. to transfer mesh associativity to the original manifold assembly, Fig. 17 (a). Topological dependencies between equivalent manifold and non-manifold representations are automatically derived using topological adjacencies in the non-manifold topology in the data structure, see [19]. Defining these relationships is essential for seamless integration between CAD and CAE disciplines, especially where distinct manifold and nonmanifold modelers tend to be utilized. Transferring mesh entities between equivalent model representations strengthens the bi-directional links required for fully coupled design and analysis tools.

Ideally the data structure described herein would interface directly with all design and analysis tools, making it easier to store the required topological links simultaneously with the ongoing design activities. However, in this work the interfacing has been prototyped using the scripting interfaces 
to certain packages. It has been shown that the links between different design and analysis geometries can be identified without having to interact with all packages in the design process. For example, it is possible to identify the links between the design and analysis geometry without having to interact with the simplification tools used to create the analysis geometry. This highlights the robustness of this approach, enabling the desired tools to be used for certain applications without having to consider the downstream effect. The memory overhead for the database is insignificant for the small models presented here. For industrial design applications the database memory overhead will depend on component complexity, the number of equivalent representations and the meshing attributes used. It is anticipated this overhead will be small relative to the corresponding CAD and CAE data files and structures.

Another method to find the Virtual Topology relationships described in Section 4 is to use the Identifier of a topological entity. If a package contains geometric searching functionality the Identifier information in the database can be used to identify entities that have been merged together. The Identifier contains the coordinates of a position within the boundary of an entity and can be used to find the closest entity located at those coordinates. A simple query on the database returns the Identifier of all edges and faces in the host model. The virtual model is interrogated to find the closest edges and faces for each Identifier. Entities in the virtual model containing multiple host Identifiers are superset entities.

In order to demonstrate the effectiveness of the proposed approach Section 6 describes how analysis geometry can be automatically linked to its equivalent original representation, allowing for robust transfer of the mesh. Once appropriate models have been created the approach is fully automated and can be used to establish the equivalence between any number of CAD or analysis models from a variety of sources. Analysis attributes have been transferred between models at various levels of fidelity within Siemens NX, Abaqus, CADFix and Parasolid. Since Parasolid is the underling modelling kernel of other CAD and CAE packages (SolidWorks, Ansys and MSC Patran), extending the current toolset to include these packages requires only implementation since the Parasolid data structure is utilized. While the analysis geometry used for the example in Figs. 14 and 15 is the output from a decomposition tool, other simplified models can also be integrated using the proposed approach. In terms of linking dimensionally reduced entities more details are provided in [9] and [19]. Once relationships have been established between dimensionally reduced cells and their equivalent original representation, mesh parentage relationships can be transferred between the models as described throughout this paper. An application to models of industrial complexity is described in [8] and [19] for equivalent decomposed and dimensionally reduced model representations. 
Robust transfer of meshes between different models and packages enables boundary conditions and other analysis attributes applied to the original model to be automatically transferred to a mesh generated on abstract analysis geometry. During automatic Virtual Topology operations there is the possibility that topological entities with boundary conditions applied may be merged with adjacent entities. It has been assumed in this work that entities with boundary conditions applied will not be involved in downstream clean-up operations. In practice it may be necessary to store the original topology in the database along with pointers to entities with boundary conditions applied to ensure these entities would be preserved. Additionally, boundary conditions can be attached to virtual entities, where for example virtual subsets may be created to apply contact between faces.

One current limitation is for mixed solid meshing and the treatment of non-conforming interface regions, i.e. hex and tet meshes meeting at a common face results in a non-conforming mesh at the interface. The distinct meshes can be coupled using multi-point constraint equations or by inserting transition elements, such as pyramids. For example, the node insertion method [12] can be used to partition hex elements at a nonconforming interface by inserting a node at the centroids of the hexes, generating two tet elements and five pyramid elements and creating conformity at the interface. The non-manifold model utilized in this work enables interface elements to be easily identified so that they can be partitioned. Once partitioned, the resulting tet and pyramid elements are considered as subsets of the original hex element. In cases where it is desirable to preserve the hex elements at the interface, conformity may be achieved by merging adjacent tet elements together. The new pyramid elements are considered virtual supersets of the original tet elements. In this manner Virtual Topology is extended to manage different decompositions of equivalent meshes, allowing multiple mesh representations to be linked to the same base geometry.

\section{Conclusions}

Novel techniques have been presented in this paper to facilitate the transfer of finite element meshes between equivalent analysis geometries residing in different CAE packages, without any loss of integrity. This is achieved by tracking the equivalences between all cells in the equivalent design and analysis models. Virtual Topology merging and partitioning are used to create a cellular decomposition of the design space, which provides a framework upon which to specify all the necessary analysis attributes.

In order to transfer the mesh between packages, the simplified analysis model is linked to its equivalent design model. Equivalent relationships are stored in a robust manner so they can be reused downstream in different 
packages, enabling mesh-geometry ownership to be transferred between the models at different levels of abstraction and fidelity. Therefore, boundary conditions applied to an original model can be automatically transferred to the finite element mesh generated on abstract analysis geometry. This enables toolsets of choice to be selected by an analyst without having to manually link models, resulting in an integrated analysis process. This integration allows analysis model pre-processing tasks (geometry clean-up and decomposition) and analysis setup (loading and boundary conditions) to be carried out concurrently.

\section{References}

1. Arabashi, S.D., Barton, C. and Shaw, N.K., "Steps towards cad-fea integration," Engineering with Computers, vol. 9, no. 1, pp. 17-26, 1993.

2. Beall, M.W. and Shephard, M.S., "Accessing CAD geometry for mesh generation," in 12th International Meshing Roundtable, Santa Fe, 2003.

3. Cavalcanti, P.R., Carvalho, P.C. and Martha, L.F., "Non-manifold modeling: An approach based on spatial subdivision," Computer-Aided Design, vol. 29, no. 3, pp. 209-220, 1997.

4. Donaghy, R.,McCune, W., Bridgett, S., Armstrong, C. G.Robinson, D.J. and McKeag, R. M., "Dimensional reduction of analysis models," in Proceedings of the 5th International Meshing Roundtable, 1996.

5. Harlin, G., "Engineering value of simulation process and data-management applied to aero engine design," in Nafems World Congress, Salzburg, 2013.

6. Lee, J.Y., Lee, J.H., Kim, H., Kim, H.S., “A Cellular topology-based approach to generating progressive solid models from feature-centric models", Computer-Aided Design, vol. 36, no. 3, pp. 217-229, 2004.

7. Lee, S.H., "A CAD-CAE integration approach using feature based multiresolution and multi-abstraction modeling techniques," Computer-Aided Design, pp. 941-955, 2005.

8. Makem, J.E., Armstrong, C.G. and Robinson, T.T., "Automatic decomposition and efficient semi-structured meshing of complex solids," Engineering with Computers, pp. DOI: 10.1007/s00366-012-0302-x, 2012.

9. Nolan, D.C., Tierney, C.M., Armstrong,C.G., Robinson,T.T. and Makem,J.E., "Automatic dimensional reduction and meshing of stiffened thin-wall structures," Engineering with Computers, DOI: 10.1007/s00366-013-0317-2013.

10. Nolan, D.C., Tierney, C.M., Armstrong,C.G., Robinson,T.T, "Automating analysis modelling though the use of Simulation Intent," 2013. Paper presented at NAFEM World Congress, Salzburg, Austria.

11. Owen, S.J. and Shepherd, J.F., "Embedding features in a Cartesian grid," in 18th International Roundtable, Salt Lake City, 2009.

12. Owen, S.J., Cannan, S.A. and Saigal, S., "Pyramid elements for maintaining tetrahedra to hexahedra conformity," ASME, pp. 123-129, 1997.

13. Robinson, T.T., Armstrong, C.G. and Fairey, R., "Automated mixed dimensional modeling from $2 \mathrm{~d}$ and $3 \mathrm{~d}$ cad models," Finite Elements in Analysis and Design, vol. 47, no. 2, pp. 151-165, 2011. 
14. Sheffer, A., Blacker, T., Bercovier, M., "Virtual Topology Operators for Meshing", International Journal of Computational Geometry and Applications, 10(3), 309-331, 2000

15. Shephard, M. S., Beall, M.W., O'Bara, R.M. and Webster, B.E., "Toward simulation-based design," Finite Elements in Analysis and Design, pp. 15751598, 2004.

16. Sypkens Smit, M and Bronsvoort, W.F., "Integration of Design and Analysis Models," Computer-Aided Design and Applications, pp. 795-808, 2009.

17. Sypkens Smit, M and Bronsvoort, W.F., "Efficient tetrahedral remeshing of feature models for finite element analysis," Engineering with Computers, vol. 25, pp. 327-344, 2009.

18. Thakur, A., Banerjee, A.G. and Gupta, S.K., "A survey of CAD model simplification techniques for physics-based simulation applications," ComputerAided Design, vol. 41, pp. 65-80, 2009.

19. Tierney, C.M., Nolan, D.C., Robinson, T.T. and Armstrong, C.G., "Managing equivalent representations of design and analysis models," Computer-Aided Design and Applications, 11:2, 193-205, DOI: 10.1080/16864360.2014.846091, 2014.

20. Weiler, K., "The Radial Edge Structure," in Geometric Modeling for CAD applications, North-Holland, 1988, pp. 3-36.

21. White, D. and Saigal, S., "Improved imprint and merge for conformal meshing," in 11th International Meshing Roundtable, New York, 2002. 